\title{
Signatures of recent star formation in ring S0 galaxies
}

\author{
A. Marino, L. Bianchi • R. Rampazzo • D. Thilker \\ - F. Annibali, A. Bressan, L.M. Buson
}

\begin{abstract}
We present a study of the stellar populations of ring and/or arm-like structures in a sample of S0 galaxies using GALEX far- and near-ultraviolet imaging and SDSS optical data. Such structures are prominent in the UV and reveal recent star formation. We quantitatively characterize these rejuvenation events, estimating the average age and stellar mass of the ring structures, as well as of the entire galaxy. The mass fraction of the UV-bright rings is a few percent of the total galaxy mass, although the UV ring luminosity reaches $70 \%$ of the galaxy luminosity. The integrated colors of these S0s locates them in the red sequence (NGC 2962) and in the so-called green valley. We suggest that the star formation episodes may be induced by different triggering mechanisms, such as the inner secular evolution driven by bars, and interaction episodes.
\end{abstract}

Keywords Galaxies: elliptical and lenticular; Galaxies: photometry; Galaxies: fundamental parameters; Galaxies: formation; Galaxies: evolution; Galaxies: Ultraviolet imaging.

\section{A. Marino, L. Bianchi}

Dept. of Physics \& Astronomy

Johns Hopkins University

3400 N. Charles St.

Baltimore, MD 21218

R. Rampazzo

INAF Osservatorio Astronomico di Padova

Vicolo dell'Osservatorio 5

I-35122 Padova, Italy

D. Thilker

Dept. of Physics \& Astronomy

Johns Hopkins University

3400 N. Charles St.

Baltimore, MD 21218

F. Annibali, A. Bressan, L.M. Buson

INAF Osservatorio Astronomico di Padova

Vicolo dell'Osservatorio 5

I-35122 Padova, Italy

\section{Introduction}

S0 galaxies have been introduced by Hubble (1936) in his "tuning fork" galaxy classification as a more or less hypothetical transition class between ellipticals and spirals. The bulge and the disk are the defining structures of S0s. Later classification schemes by de Vaucouleurs et al. (1991, RC3 hereafter) and Sandage \& Tammann (1987, RSA hereafter) take into account the presence of the several sub-structures detected within the S0s class: bars, inner and outer rings, as well as lenses (from here the widely used term of lenticulars as synonym of S0s) and ovals are often found.

S0s are, as a rule, admitted in the vast class of earlytype galaxies (ETGs hereafter), together with Ellipticals, with which they share typically passively evolving stellar populations. From an evolutionary point of view it is widely believed that S0s were initially spirals which lost their interstellar medium (ISM hereafter) during collisions (Spitzer \& Baade 1951) by "harassment" (Moore et al. 1996) or by "ram-pressure". Simulations show that such events could occur both in clusters (Gunn \& Gott 1972) and in groups (see e.g. Bekki 2009).

From the kinematical point of view, genuine S0s are quite distinct from giant ellipticals since they are fast rotating, like late-type galaxies. At the same time, if S0s were originally spirals, they should have formed their mass over a significant fraction of the Hubble time, following a star formation history more similar to that of late-type galaxies rather than that of giant elliptical galaxies. In this context, our multi-wavelength study of nearby ETGs (Marino et al. 2010a) shows that S0s, characterized through their luminosity profiles and by the low values of the $n$ index of the Sersic law Sersic 1968), have the lowest values of the $\alpha$-enhancements $([\alpha / F e])$ in the sample. The low $[\alpha / F e]$ values suggest a "more prolonged" star formation in the S0s galaxies 


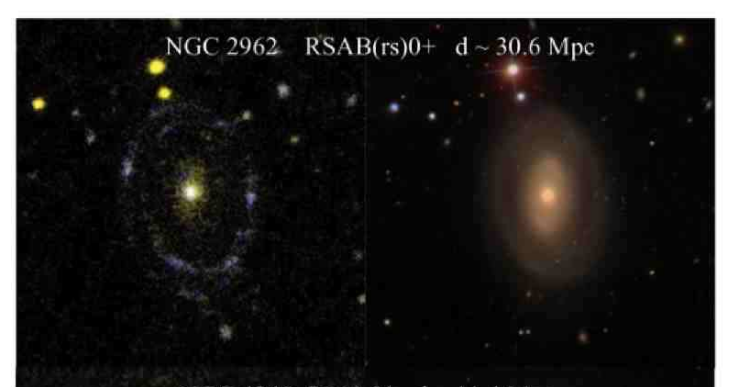

NGC $4245 \mathrm{SB}(\mathrm{r})$ 0/a d 11.4 Mpc
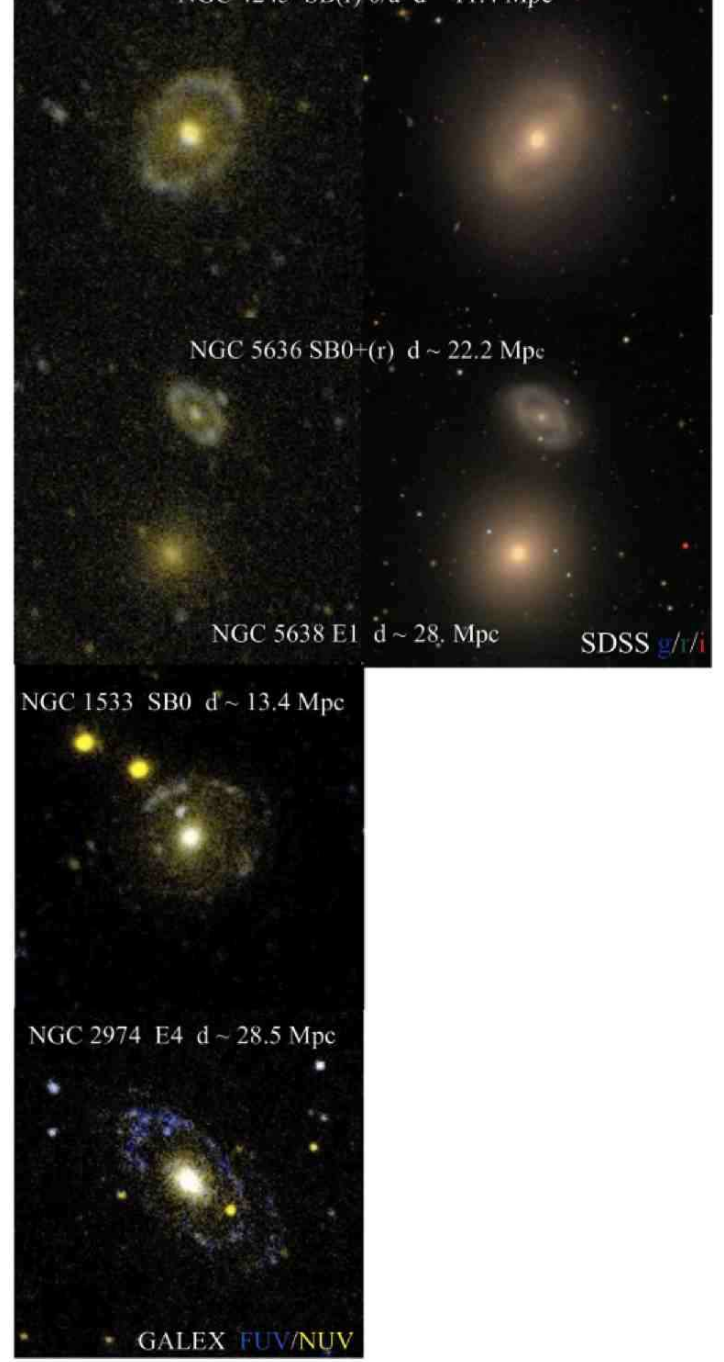

Fig. 1 Composite GALEX images (FUV=blue and $\mathrm{NUV}=$ yellow; left panels) and $\operatorname{SDSS}(g=$ blue, $r=$ green, $i=$ red; right panels) color composite images of the $\mathrm{S} 0$ galaxies NGC 1533, NGC 2962, NGC 2974, NGC 4245 and NGC 5636 and of the elliptical NGC 5638. The outer ring structures appear in the UV less smooth than in optical (in spite of the SDSS higher resolution) and bluer than the nucleus, suggesting the presence of young stellar populations with respect to ellipticals in the sample Annibali et al. 2007, 2010, see also Rampazzo et al. these proceedings).

Several mechanisms, both internal and external to the galaxy, may be envisaged to produce the signature of a "prolonged" star formation. The removal of the ISM from a possible spiral precursor may quench the ongoing star formation transforming the debris in an S0 with signatures of prolonged star formation, as well as an external "wet" accretion and/or the inner secular evolution, e.g. driven by a tumbling bar (see e.g. van den Bosch \& Emsellem 1998).

The Galaxy Evolution Explorer satellite (GALEX hereafter) has widely contributed to explore the above mechanisms. "Wet" accretions, and the rejuvenation of the stellar population, has been evidenced in ETGs (see e.g. Marino et al. 2009, and Rampazzo et al. in these proceedings). Recently, Thilker et al. (2010) found in NGC 404, a nearby well known S0, an external ringlike structure with signature of recent star formation. $70 \%$ of the FUV comes from an HI ring forming stars at a rate of $2.5 \times 10^{-3} M_{\odot} \mathrm{yr}^{-1}$. The structure has been likely produced by a "wet accretion/merger". Along this line, using GALEX we analyze five S0s, showing outer rings and/or arm-like structures, from the sample of Marino et al. (2010a , c) aiming at understanding the nature of the ring and to map possible star formation episodes.

\section{The sample}

The sample includes five S0 galaxies: NGC 1533, NGC 2962, NGC 2974, NGC 4245 and NGC 5636 (interacting with NGC 5638, an elliptical galaxy). Notice that NGC 2974 is erroneously classified as elliptical both in RC3 and RSA, although an exponential disk is evident from the optical and UV luminosity profiles (see also Jeong et al. 2007, 2009; Marino et al. 2010a). In addition, in NGC 2974 Krajnović et al. (2005) found, with SAURON observations (de Zeeuw et al. 2002), the existence of non-axisymmetric perturbations consistent with the presence of inner bars. Summarizing, all galaxies in the present sample are barred S0s.

In some of these galaxies the literature reports signatures of both possible recent accretion events and of ongoing interaction. Tal et al. (2009) report the presence of a shell system surrounding NGC 2974, a signature of a minor merging event (Dupraz \& Combes 1986; Ebrova et al. 2009). Werk et al. (2010) detected a large HI structure (see her Figure 7) extending for several kiloparsec outside NGC 1553 towards the interacting companion, IC 2038. In the HI cloud, well outside the NGC $1553 \mathrm{ring}$, OB associations are also 

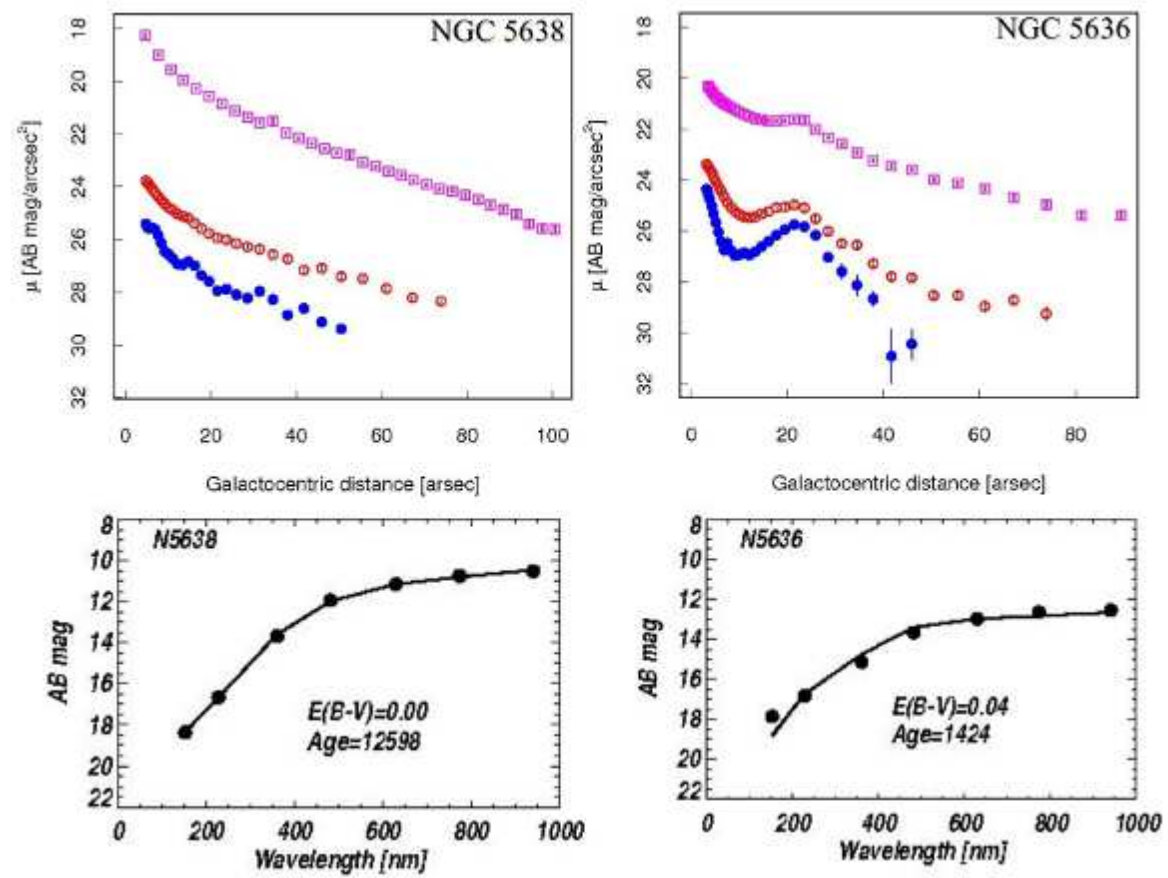

Fig. 2 In the top right panel the optical and UV surface brightness profiles of NGC 5636 are plotted. Blue filled circles represent FUV, empty red circles NUV, and magenta empty squares SDSSr. For comparison, the smooth FUV, NUV and $r$ surface brightness profiles of the elliptical NGC 5638 are shown in the top left panel. In the bottom panels the full dots represent the FUV, NUV, $u, g, r, i, z$ spectral energy distribution of these galaxies. Best fit models, assuming only foreground extinction, are indicated with continuous lines (see text). Ages, derived from SED fitting, are reported in Myrs

found. DeGraaff et al. (2007) describes this galaxy as a late stage of transition from a barred spiral to a barred S0 galaxy.

All S0s belong to low density environments since they are members of loose groups of galaxies. NGC 1533 is member of the Dorado group. NGC 2962, NGC2974, NGC 5636 and its elliptical companion NGC 5638, belong to LGG 178, LGG 179, LGG 386 groups, respectively (Garcia 1993). NGC 4245 is located in the spiral-dominated group USGC-U478 (Ramella et al. 2002).

\section{Observations}

UV imaging was obtained with GALEX (Martin et al. 2005; Morrissey et al. 2007) in far-Ultraviolet (FUV, $1344-1786 \AA$ ) and near-Ultraviolet (NUV, $1771-2831$ $\AA$ ) bands. The journal of the GALEX observations is given in Table 1. In Figure 1 we show the composite UV and optical images of the galaxies.

Ancillary optical data for NGC 2962, NGC 4245, NGC 5636 were also retrieved from the SDSS archive (Adelman et al. 2008) in the $u$ [2980-4130 $\mathrm{A}$ ], g [3630-
$5830 \AA], r[5380-7230 \AA], i[6430-8630 \AA]$ and $z$ [7730$11230 \AA ̊]$ bands.

Table 1 Journal of the GALEX observations

\begin{tabular}{llll}
\hline \hline Ident. & $\begin{array}{l}\text { FUV } \\
\text { Expos. } \\
\text { [sec] }\end{array}$ & $\begin{array}{l}\text { NUV } \\
\text { Expos. } \\
{[\mathrm{sec}]}\end{array}$ & $\begin{array}{l}\text { Observing } \\
\text { program }\end{array}$ \\
\hline NGC 1533 & 1520 & 3152 & GI3 087004 \\
NGC 2962 & 2297 & 2297 & MISWZN09_24136_0336 \\
NGC 2974 & 2657 & 2657 & GI1_109006 \\
NGC 4245 & 1680 & 1680 & GI3_041006_NGC4245_0001 \\
NGC 5636 & 1704 & 1704 & MISDR1_33739_0535 \\
\hline
\end{tabular}

\section{The UV-bright outer rings}

NGC 1533 UV image shows an incomplete, clumpy ring-like structure, while NGC 2962, NGC 4245 and NGC 5636 which have well defined and complete rings, although not uniform, in the FUV and NUV bands. Spiral arm-like structures are visible in both NGC 2962 and in NGC 2974. Both the ring and the arm-like structure appear bluer than the nucleus and the galaxy body. The bar present in the optical images of the galaxies are barely visible in the FUV and NUV images. In all 
but NGC 2962 and NGC 5636 rings are within the $D_{25}$ isophote.

In Figure 2(top panel) we show the FUV, NUV and $r$ bands surface brightness profiles of NGC 5636 and, for comparison, the smooth profiles of the elliptical companion NGC 5638, obtained with the ELLPISE routine in the STSDAS package of IRAF. Rings produce a hump in the surface brightness profiles, more prominent in FUV and NUV than in optical. Outer rings are bluer than the body of their respective galaxies indicating the presence of young stellar populations.

We compute FUV, NUV and optical magnitudes of the outer ring structures using surface brightness profiles after subtracting the galaxy contribution as described by Marino et al. (2010b). About 25\%, 71\%, $30 \%, 33 \%$ and $60 \%$ of the total FUV luminosity of NGC 1533, NGC 2962, NGC 2974, NGC 4245, NGC 5636 comes from the ring structure.

We also compute the total galaxy magnitude in the $\mathrm{AB}$ system integrating the sky-subtracted UV and optical galaxy light within concentric elliptical apertures enclosing the galaxy. This aperture photometry provides the FUV to near-Infrared spectral energy distribution (SED hereafter) of our galaxies. In Figure 2 we plot, as an example, the SEDs of NGC 5636 and of the companion elliptical galaxy NGC 5638 (see next section).

\section{Characterizing the star formation history}

We aim at characterizing the strength and the epoch of the rejuvenation episodes in S0s by estimating the ages and stellar masses of the outer rings and of the entire galaxy.

To this purpose, we compare the observed SEDs with population synthesis models computed with the GRASIL code (Silva et al. 1998) which takes into account the effects of the internal extinction. The star formation history (SFH) and the Initial Mass Function (IMF), the metallicity and the residual gas fraction are given as input parameters of the code. We adopted two different SFHs: one typical of elliptical galaxies, characterized by a short (1 Gyr) and intense period of star formation followed by pure passive stellar evolution, and the other one, typical of spirals with a more prolonged SF. We used a Salpeter IMF with a mass range between 0.15 and $120 \mathrm{M}_{\odot}$ for ellipticals and between $0.1-100$ $\mathrm{M}_{\odot}$ for spirals following Silva et al. (1998). A gas time infall $t_{\text {inf }}=0.1$ and 4 Gyr and a star formation efficiency $\nu=2$ and 0.6 have been used for elliptical and spirals respectively. The code computes the integrated spectrum taking into account the stellar and gas budget at any

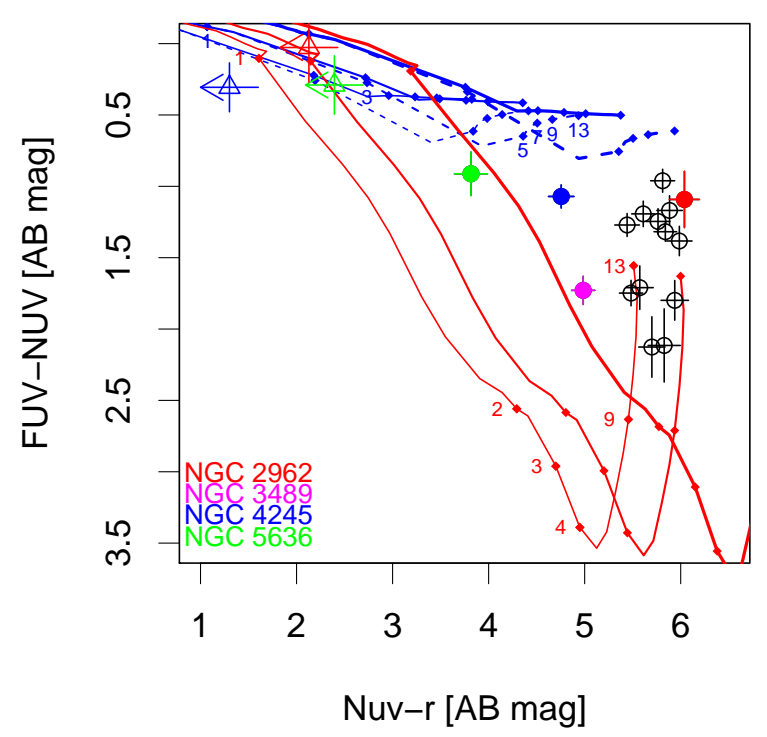

Fig. 3 Top: $(\mathrm{NUV}-r)$ vs (FUV-NUV) measurements of outer ring structures (triangles), and total (colored filled circles) of NGC 2962, NGC 5636, NGC 4245. We also plot NGC 3489, an S0 in the sample of Marino et al. (2010a), showing blue arm-like structures. Black open circles plot ETGs in Marino et al. (2010a) without ring structures. Blue lines represent GRASIL model colors for disk-type SFH with different extinction $(\mathrm{E}(\mathrm{B}-\mathrm{V})=0,0.1,0.3$ from thin to thick lines) and inclination (solid line $\mathrm{i}=0^{\circ}$, dashed line $\mathrm{i}=90^{\circ}$ ). Red lines are GRASIL model colors for E-type SFH (passive evolution). The UV-rising part in E-type models at old ages is due to post-AGB stars. Numbers on the model lines are ages in Gyrs

age. It includes the effect of the age-dependent extinction with young stars being more affected by dust.

In Figure 2 (bottom panels) we plot SEDs of NGC 5636 and NGC 5638, obtained integrating the light of the entire galaxy, and the best-fit model obtained adopting an Elliptical SFH.

The elliptical NGC 5638 is well fitted with an old age (12 Gyr) of the stellar population. The case of the ring S0 companion NGC 5636 is quite different: the observed SED shows a FUV excess with respect to the best-fit model assuming foreground extinction of 0.04 indicating that a simply passive $\mathrm{SFH}$ is inappropriate. Ages and stellar masses of the outer ring structures were estimated using FUV and NUV measurements because only upper limits can be derived from the SDSS images. For the ring structures we derive very young ages $(\lesssim$ 200 Myr) adopting a Single Stellar population (SSP), and $\sim 1$ Gyr with an Elliptical SFH while assuming 
Spiral SFH ages are between 2 and 4 Gyr. The stellar mass of the outer rings is about $1-4 \%, 1 \%, 1 \%$ and $5-8 \%$ of the total stellar mass of NGC 1533, NGC 2962, NGC 2974, NGC 4245, NGC 5636, respectively (details are provided in Marino et al. 2010b.

Combining the UV and the SDSS photometry we plot in Figure 3 the (FUV-NUV) vs. (NUV-r ) color diagram for ring structures (triangles), the total S0 ring galaxies (full dots) and ETGs without ring (open circles) from Marino et al. (2010a). In the (FUV-NUV) vs. (NUV-r) plane rings and entire galaxies appears well separated in color. The two sets of GRASIL models are also shown to help in interpreting the data. The color of the rings are compatible either with models for a continuous SFH (blue lines) or with the SFH adopted for elliptical (red lines) but in the very young $(<1$ Gyr) phase. The global colors of the galaxies plotted in the figure are affected by the younger (bluer) rings so that, on the average, the galaxy appears in all rejuvenated, in particular the cases of NGC 5636 and NGC 4245. Notice, however, that a large fraction of the ETGs from the Marino et al. (2010a) sample appear quite old objects.

In Figure 4 we plot the (NUV-r) vs. $\mathrm{M}_{r}$ color magnitude relation for our galaxies and the sample of ETGs in Marino et al. (2010a). ETGs define, with a large dispersion, the red sequence in this plane. S0s with rings like NGC 4245 and NGC 5636 are located in the so called green valley, where evolving objects are found, while the ring structures are located in the blue sequence.

\section{Star Formation triggering mechanisms}

The blue, star forming rings in NGC 4245, NGC 5636 and NGC 1533 are located at the end of the bar structure. The optical and UV images, shown in Figure 1, suggests that NGC 2962 has two rings, one at the edge of the bar, the other is an outer ring. Only the outer ring in NGC 2962 is visible in UV GALEX images. The structure of the ring and the outer features detected in NGC 2974 are reminiscent of the NGC 2962 case, which is seen more face-on. We remind the presence of a shell structure in NGC 2974 revealed by Tal et al. (2009). Shell structures suggest the occurrence of an accretion phenomenon.

According to different statistical estimations, the outer rings are observed in a significant fraction, up to $20-30 \%$, of spiral and lenticular galaxies and are closely associated with the non-axisymmetric structures like bars, ovals or triaxial bulges. Distinct ring structures are often found in the same galaxy (Emsellem et al.

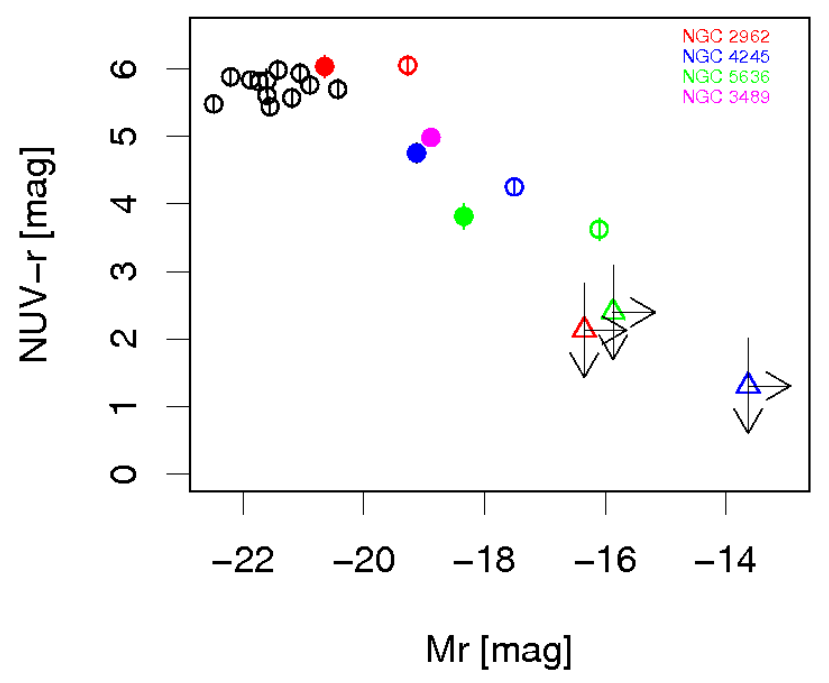

Fig. $4 M_{r}$ vs $(N U V-r)$ measurements of the outer ring structures only (triangles), of the central portion (empty circles) and of the total extent (filled circles) of NGC 2962 (red), NGC 5636 (green), NGC 4245 (blue) and NGC 3489 (magenta). Black open circles plot ETGs in Marino et al. (2010a) without ring structures. NGC 2962 is in the red sequence (e.g. Salim et al. 2007) while rings lie the blue sequence. NGC 3489, NGC 4245 and NGC 5636 lie in the so called green valley

1996). In terms of the theory, the empirical subdivision of the rings into nuclear, outer and inner corresponds to the inner, outer Lindblads and ultraharmonic dynamical resonances between the epicyclic oscillations in the stellar component and rotation of the bar (see the kinematic review of Moiseev \& Bizyaev 2009). The literature reports cases of rings without bar which are explained by weakly triaxial distortions, or by tidal actions from a gravitationally bounded companion, since the non-axisymmetric gravitational perturbations in these cases are similar to those from a bar (Moiseev \& Bizvaev 2009).

Although NGC 2974 may have suffered an accretion event, we suggest that rings in our barred S0s sample are an effect of the secular (inner) evolution (see also Jeong et al. 2007). The tumbling bar redistributes gas angular momentum, and the gas accumulates at the Lindblad's resonances (see a review by Buta \& Combes 1996) where the star formation may take place. Notice that in NGC 1533 the HI is located outside the galaxy ring: the masses of the outer rings do not require a large gas supply, and are consistent with these strcutures having formed from mass loss by the older population of stars (van den Bosch \& Emsellem 1998).

Kinematics observations will be crucial to fully characterize the phenomenon. 
Acknowledgements A. Marino acknowledges support from the Italian Scientist and Scolar of North America Foundation (ISSNAF) through an ISSNAF fellowship in Space Physics and Engineering, sponsored by Thales Alenia Space. RR acknowledges financial support from the agreement ASI-INAF I/009/10/0. GALEX is a NASA Small Explorer, launched in April 2003. GALEX is operated for NASA by California Institute of Technology under NASA contract NAS98034. IRAF is distributed by the National Optical Astronomy Observatories, which are operated by the Association of Universities for Research in Astronomy, Inc., under cooperative agreement with the National Science Foundation. This research has made use of SAOImage DS9, developed by Smithsonian Astrophysical Observatory and of the NASA/IPAC Extragalactic Database (NED) which is operated by the Jet Propulsion Laboratory, California Institute of Technology, under contract with the National Aeronautics and Space Administration. We acknowledge the usage of the HyperLeda database (http://leda.univ-lyon1.fr).

\section{References}

Adelman-McCarth, J.K., Agüeros, M.A., Allam, S.S., Allende Prieto, C., Anderson, K. S. J. et al. 2008, Astrophys. J. Suppl. Ser., 175, 297

Annibali, F., Bressan, A., Rampazzo, R., Zeilinger, W.W., Danese, L. 2007, Astron. Astrophys., 463, 455

Annibali, F., Bressan, A., Rampazzo, R., Zeilinger, W.W., Vega, O., Panuzzo, P. 2010, Astron. Astrophys., 519, A40

Bekki, K. 2009, Mon. Not. R. Astron. Soc., 399, 2221

Buta, R., Combes, F. 2009, Galactic Rings, Fundamentals of Cosmic Physics 17, 95.

Garcia, A.M. 1993, Astron. \& Astrophys. Supplement Series, 100, 47

DeGraaff, R.B., Blakeslee, J.P., Meurer, G.R., Putman, M.E., 2007, Astrophys. J., 671, 1624

de Vaucouleurs, G., de Vaucouleurs, A., Corwin, H.G. Jr. et al. 1991 Third Reference Catalogue of Bright Galaxies, Springer-Verlag, New York

de Zeeuw P.T., Bureau M., Emsellem E., Bacon R., Carollo C.M., Copin Y., Davies R.L., Kuntschner H., Miller B.W., Monnet G., Peletier R.F., Verolme E.K., 2002, Mon. Not. R. Astron. Soc., 329, 513

Dupraz,C., Combes, F. 1986, Astron. Astrophys., 166, 53

Ebrovà, I., Jungwiert, B., Canalizo, G., Bennert, N., Jilkov`a, L. 2009, in Galaxy Wars: Stellar Populations and Star Formation in Interacting Galaxies edited by Beverly Smith, Nate Bastian, Sarah J. U. Higdon, and James L. Higdon, ASP Conference Series Vol. 423, 236

Emsellem, E., Bacon, R., Monnet, G., Poulian, P. 1996, Astron. Astrophys., 312, 777

Krajnović, D., Cappellari, M., Emsellem, E., McDermid, R.M., de Zeeuw, T.P. 2005, Mon. Not. R. Astron. Soc., 357,1113
Hubble, E.P. 1936, The Realm of the Nebulae, Yale Univ. Press

Gunn, J. E., Gott, J. R.III, 1972, Astrophys. J., 176, 1

Jeong, H., Bureau, M., Yi, S. K., Krajnović, D., \& Davies, R. L. 2007, Mon. Not. R. Astron. Soc., 376, 1021

Jeong, H., Yi, S., Bureau, M., Davies, R., Falcón-Barroso, J. et al. 2009, Mon. Not. R. Astron. Soc., 398, 2028

Marino, A., Iodice, E., Tantalo, R., Piovan, L., Bettoni, D., et al. 2009, Astron. Astrophys., 508, 1235

Marino, A., Rampazzo, R., Bianchi, L., Annibali, F., Bressan, A., Buson, L.M., Clemens, M.S., Panuzzo, P., Zeilinger, W.W 2010a, Mon. Not. R. Astron. Soc., in press, (DOI: 10.1111/j.1365-2966.2010.17684.x)

Marino, A., Bianchi, L., Rampazzo, R., et al. 2010b, ApJ, submitted

Marino, A., Bianchi, L., Rampazzo, R., et al. 2010c, Astron. Astrophys., in preparation

Martin, D.C., Fanson, J., Schiminovich, D. et al. 2006, ApJ, 619, L1

Moiseev, A.V. Bizyaev, D.V. 2009, New Astron.Rev., 53, 169

Morrissey, P., Conroy, T., Barlow, T.A., et al. 2007, ApJS, 173,682

Moore, B. Katz, N., Lake, G., Dressler, A., Oemler, A. 1986, Nature, 379, 613

Ramella, M., Geller, M.J., Pisani, A., da Costa, L.N. 2002, Astron. J., 123, 2976

Rampazzo, R., Marino, A., Tantalo, R., Bettoni, D., Buson, L. M., Chiosi, C., Galletta, G., Grützbauch, R., Rich, R. M. 2007, Mon. Not. R. Astron. Soc., 381, 245

Salim, S., Rich, R. M., Charlot, S., Brinchmann, J., Johnson, B. D., Schiminovich, D. et al. 2007, Astrophys. J. Suppl. Ser., 173, 267

Sandage, A.R., Tammann, G. 1987, A Revised Shapley Ames Catalogue of Bright Galaxies, Carnegie, Washington (RSA)

Sersic, J. L. 1968, Atlas de Galaxias Australes, Osservatorio Astronomico, Cordoba

Silva, L., Granato, G.L., Bressan, A., Danese, L. 1998, ApJ, 509, 103

Schawinski, K., Kaviraj, S., Khochfar, S., Yoon, S.-J., Yi, S. K. et al. 2007, Astrophys. J. Suppl. Ser., 173, 512

Spitzer, L.Jr., Baade, W. 1951, Astrophys. J., 113, 413

Tal., T., van Dokkum, P.G., Nelan, J., Bezanson, R. 2009, Astron. J., 138, 1417

Thilker, D.A., Bianchi, L., Schiminovic, D. et al. 2010, Astrophys. J., 714, L171

van den Bosch, F.C., Emsellem, E. 1998, Mon. Not. R. Astron. Soc., 298, 267

Werk, J.K., Putman, M.E., Meurer, G.R. et al. 2010, Astron. J., 139, 279

This manuscript was prepared with the AAS LATEX macros v5.2. 